\title{
Surface Motion Graphs for Character Animation from 3D Video
}

\author{
Peng Huang* and Adrian Hilton \\ Centre for Vision Speech and Signal Processing \\ University of Surrey, UK, GU2 7YW
}
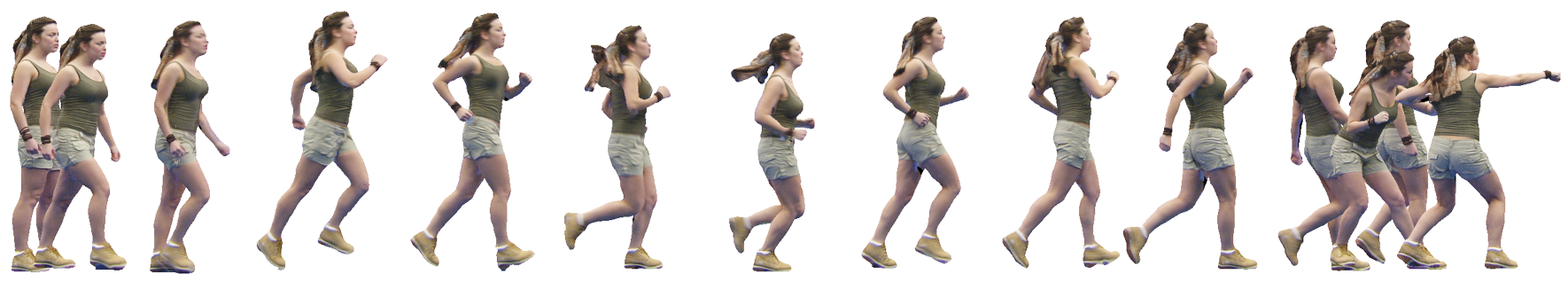

Figure 1: An example of synthesized 3D character animation (10 transitions). Target: Stand\#1 $\rightarrow$ Hit\#45, 10 metres, 250 frames.

\section{Introduction}

Multiple view reconstruction of human performance as a 3D video has advanced to the stage of capturing detailed non-rigid dynamic surface shape and appearance of the body, clothing and hair during motion [Aguiar et al. 2008; Starck and Hilton 2007]. Full 3D video scene capture holds the potential to create truly realistic synthetic animated content by reproducing the dynamics of shape and appearance currently missing from marker-based motion capture. However, the acquisition results are in an unstructured volumetric or mesh approximation of the surface shape at each frame without temporal correspondence, which makes the reuse of this kind of data more challenging than conventional mocap data. In this paper, we introduce a framework that automatically constructs motion graphs for 3D video sequences and synthesizes novel animations to best satisfy user specified constraints on movement, location and timing.

\section{Methodology}

The framework comprises two stages: pre-processing the database of the 3D video sequences into a surface motion graph; and motion synthesis by optimizing the graph-path to satisfy user-defined constraints and minimize the transition cost. The surface motion graph represents the possible transitions which is analogous to motion graphs introduced by [Kovar et al. 2002] for skeletal motion capture sequences. Skeletal motion capture data has known temporal correspondence allowing the definition of similarity metrics to identify transitions that will not cause unnatural motion. Accurate estimation of temporal surface correspondence for 3D video sequences of human motion is an open-problem [Vlasic et al. 2008; Aguiar et al. 2008]. Current approaches are computationally expensive and require manual intervention to obtain correct correspondences for complex non-rigid surface motion. In this paper, transition points between 3D video sequences are identified without temporal correspondence using a volumetric temporal shape similarity metric which measures similarity in both $3 \mathrm{D}$ shape and motion [Huang et al. 2007].

The database of 3D video sequences is structured as a two-level directed surface motion graph (SMG). In the higher-level, each node represents a motion and each edge a transition. In the lower-level, each node represents a frame serving as a possible transition point and each edge a sequence of frames connecting them. The cost of a path through the surface motion graph is defined according to the total transition cost $C_{s}$, location cost $C_{d}$ and time cost $C_{t}$. The optimal path minimizes this cost. Given a path $F$ the total distance, time and smoothness are computed independently and combined together as a weighted sum. The optimal path $F_{\text {opt }}$ can be found by minimizing the combination:

$$
F_{\text {opt }}=\underset{F}{\arg \min }\left\{C_{s}(F)+w_{d} C_{d}(F)+w_{t} C_{t}(F)\right\}
$$

where $w_{d}$ and $w_{t}$ are weights for distance and time constraints respectively. The path $F$ can be decomposed into paths without loops $l_{0}$ between the start and end key-frame and a set of loops for each path $\left\{l_{i}\right\}, i=1 . . N_{l}$. The task then becomes to find an optimal set of integer number $\left\{n_{i}\right\}_{\text {opt }}$ corresponding to repetitions of loops which minimizes (1):

$$
\left\{n_{i}\right\}_{o p t}=\underset{\left\{n_{i}\right\}}{\arg \min } \sum_{i=0}^{N_{l}}\left\{C_{s}\left(l_{i}\right)+w_{d} C_{d}\left(l_{i}\right)+w_{t} C_{t}\left(l_{i}\right)\right\} \cdot n_{i}
$$

This optimisation can be solved efficiently as an Integer Linear Programming problem. Results demonstrate that concatenative synthesis of novel sequences accurately satisfy the user constraints and produce motions which preserve the detailed non-rigid dynamics of clothing and loose hair. Figure 1 shows an example of synthesized 3D character animation from a public available database of 3D video [Starck and Hilton 2007] using view-dependant rendering.

\section{References}

Aguiar, E., Stoll, C., Theobalt, C., Ahmed, N., Seidel, H.-P., And Thrun, S. 2008. Performance capture from sparse multi-view video. ACM Trans. Graph. $27,3,1-10$.

Huang, P., Starck, J., And Hilton, A. 2007. A study of shape similarity for temporal surface sequences of people. In 3DIM '07: Proceedings of the Sixth International Conference on 3-D Digital Imaging and Modeling, IEEE Computer Society, Washington, DC, USA, 408-418.

Kovar, L., Gleicher, M., AND Pighin, F. 2002. Motion graphs. In SIGGRAPH '02: Proceedings of the 29th annual conference on Computer graphics and interactive techniques, ACM Press, New York, NY, USA, vol. 21, 473-482.

StaRCK, J., AND HiLton, A. 2007. Surface capture for performance-based animation. IEEE Computer Graphics and Applications 27, 3, 21-31.

Vlasic, D., Baran, I., Matusik, W., AND Popović, J. 2008. Articulated mesh animation from multi-view silhouettes. ACM Trans. Graph. 27, 3, 1-9.

*e-mail: p.huang@surrey.ac.uk 